\title{
Das ärztliche Preissystem: Zentrale Problemzone der ärztlichen Vergütung
}

\section{PASCAL KAISER}

Pascal Kaiser ist Referent in der Abteilung Ambulante Versorgung beim GKVSpitzenverband in Berlin

Seit Jahren steigt die ärztliche Vergütung kontinuierlich an und dennoch beklagen die Ärztevertreter die aus ihrer Sicht ungenügende Anpassung des Orientierungswertes. Im Mittelpunkt der jährlichen Vergütungsverhandlungen zwischen Kassenärztlicher Bundesvereinigung und GKV-Spitzenverband steht auf der Bundesebene der Orientierungswert. Die konkrete Umsetzung im Bewertungsausschuss wird zukünftig darüber entscheiden, wie effizient und effektiv die Preissetzung bei vertragsärztlichen Leistungen sein wird. Wie aber funktioniert das vertragsärztliche Preissystem eigentlich und wie kann es zukünftig weiterentwickelt werden? Der folgende Beitrag erläutert die Funktionsweise, klärt die Missverständnisse über das Preissystem auf und diskutiert Lösungsmöglichkeiten.

\section{Einleitung}

Die Entscheidung über die Anpassung des Orientierungswertes für das Jahr 2015 steht im Bewertungsausschuss in Kürze an. Die Verhandlungen zum Orientierungswert 2013 hatten zu heftigen Auseinandersetzungen geführt. Seitdem ist es ruhiger um die jährlichen Anpassungen geworden, aber ein konsistentes Preisermittlungsverfahren ist noch nicht in Sicht. Gleichfalls halten sich hartnäckig viele Fehlinterpretationen in der Ärzteschaft wie die Mär vom Preisverfall der ärztlichen Leistungen und entsprechenden Einkommensverlusten. So hat die $\mathrm{KBV}^{1}$ vor Kurzem in einem Positionspapier behauptet, sie würde in den Verhandlungen zum Orientierungswert ständig benachteiligt werden und die ärztlichen Einkommen würden sich unterhalb der Inflationsrate entwickeln. Tatsächlich halten diese Aus- sagen einer näheren Betrachtung nicht stand, da sie die unterschiedlichen Aspekte der Preisbestimmung miteinander vermischen. Um die Hintergründe besser nachvollziehen zu können, sollen im Folgenden die Funktionszusammenhänge des gegenwärtigen Preissystems beschrieben sowie dessen Defizite und mögliche Lösungsansätze dargestellt werden.

\section{Von der Einführung im Jahr 2009 bis heute}

Das ärztliche Vergütungssystem ist 2009 durch das GKV-Wettbewerbsstärkungsgesetz (GKV-WSG) umgestaltet worden. ${ }^{2}$ Mit der Einführung des Orientierungs-

\footnotetext{
* Der Beitrag gibt die persönliche Meinung des Autors wieder.

1 KBV (2014)

2 Zu den Hintergründen und der Bewertung der letzten Honorarreformen: Kaiser (2014)
} 
wertes gemäß $\$ 87$ Absatz 2e SGBV war das Ziel verbunden, erstens den Flickenteppich von floatenden Preisen, die sich zwischen den Kassenärztlichen Vereinigungen (KV) und auch noch quartalsweise innerhalb einer KV unterschieden, zu vereinheitlichen und zweitens für Ärzte und für Krankenkassen vergleichbare Leistungspreise und eine größere Planungssicherheit zu erreichen.

Mit dem im Bewertungsausschuss jährlich bis zum 31.8. für das folgende Jahr zu vereinbarenden Orientierungswert werden alle Punktleistungen im EBM bewertet. Er ist Grundlage für die Vereinbarung des regionalen Punktwertes, der für die Vergütung der vertragsärztlichen Leistungen heranzuziehen ist. ${ }^{3}$

Erstmals wurde der Orientierungswert für das Jahr 2009 mit 3,5001 Cent ermittelt; er war damit bezogen auf die gesamte ärztliche Leistungsmenge eigentlich überbewertet, was bis heute zu Fehlinterpretationen führt. Dies ist darauf zurückzuführen, dass der Orientierungswert aus der Gesamtvergütung $2009^{4}$ geteilt durch die Leistungsmenge (in Punkten) errechnet wurde, wobei allerdings nicht die gesamte abgerechnete Leistungsmenge (Bruttoleistungsmenge) herangezogen wurde, sondern nur die durch die jeweiligen regionalen Honorarverteilungsregelungen vergütete Leistungsmenge (Nettoleistungsmenge); diese umfasste aber nur ca. 90\% der Bruttoleistungsmenge, während die restlichen Leistungen, also ca. $10 \%$, mit einem deutlich geringeren Preis (Restpunktwert) vergütet wurden. Diese Kalkulation war durchaus im Interesse der KBV, die einen möglichst hohen Orientierungswert vereinbaren wollte, der aber bei Einbeziehung der Bruttoleistungsmenge statt bei 3,5 Cent je Leistungspunkt nur bei ca. 3,2 Cent gelegen hätte. Wenn die Ärzteseite ${ }^{5}$ also fordert, endlich alle Leistungen mit dem vollen Preis zu bezahlen, dann setzt sie sich auch dem Vorwurf der Scheinheiligkeit aus, da dies eigentlich eine Absenkung des Orientierungswertes zur Folge haben müsste.

Zwischen 2010 und 2012 wurde der Orientierungswert aufgrund der Entscheidungen im Erweiterten Bewertungsausschuss und durch das GKVFinanzierungsgesetz (GKV-FinG) nicht angepasst. Die darauf folgenden Verhandlungen im Sommer 2012 gestalteten sich von Anfang an außerordentlich schwierig. Bereits im März 2012 wur- den von der KBV Honorarsteigerungen in Höhe von 3,5 Mrd. Euro bzw. 12\% für die Orientierungswertanhebung gefordert, und dabei wäre es nicht geblieben, denn hinzuzurechnen waren noch die regionalen Morbiditätsraten und die durch das GKV-Versorgungsstrukturgesetz (GKV-VStG) erzeugten finanziellen Mehrbelastungen durch neue regionale Vergütungsregelungen. Um ein finanziell tragbares Verhandlungsergebnis erzielen zu können, musste der GKV-Spitzenverband eine angemessene Gegenforderung einbringen. Der vom Erweiterten Bewertungsausschuss schließlich gefasste Beschluss mit einer Anpassung in Höhe von 0,9\% für das Jahr 2013 wurde von der KBV, nachdem sie sich mit ihren Forderungen innerhalb der Ärzteschaft bereits festgelegt hatte, nicht akzeptiert, und sie verweigerte daraufhin die weitere Mitwirkung im Bewertungsausschuss. Der im Oktober 2012 erzielte Kompromiss bestätigte zwar die Orientierungswertanpassung, verlangte aber auch der Kassenseite zusätzliche Ausgabenerhöhungen ab. Seitdem ist es im Bewertungsausschuss gelungen, strittige Aspekte im Rahmen eines vereinbarten Verfahrens zur Festlegung des Orientierungswertes zu lösen und auf öffentlichkeitswirksame Forderungen in Milliardenhöhe zu verzichten. Die Anpassung des Orientierungswertes für das Jahr 2014 um 1,3\% $\%^{6}$ fand dementsprechend recht geräuschlos statt.

\section{Die Funktionsweise des ärztlichen Preissystems}

Die Preisbestimmung ist in jedem Wirtschaftssystem ein zentrales Problem. Preise sollen die effektive Nachfrage nach Gütern mit einer wirtschaftlichen Bereitstellung möglichst optimal verbinden. Sie stellen daher Signale für die jeweiligen Wirtschaftssubjekte dar. Da dies gerade im Gesundheitsmarkt nicht befriedigend funktioniert, wurden regulative Mechanismen eingeführt, die eine qualitativ hochwertige und effiziente Bereitstellung von Angeboten gewährleisten sollen. Die vertragsärztliche ambulante Versorgung ist in diesem Sinne ein Wirtschaftsbereich, bei dem durch staatliche Rahmenvorgaben die korporatistisch organisierten Vertragspartner, die Kassenärztlichen Vereinigungen und die gesetzlichen Krankenkassen, die Preise aushandeln. Diese Aushandlung von effizienten und dabei versorgungsorientierten Preisen sowie Leistungsmengen ist allerdings komplex und kann zu einer Reihe von Fehlinterpretationen führen. Ziel eines ärztlichen Preissystems sollte die Deckung der Kosten einer wirtschaftlich arbeitenden Arztpraxis bezogen auf die erstellten medizinisch notwendigen Leistungen sein. Geht man davon aus, dass zum Ausgangszeitpunkt eine Kostendeckung in einer wirtschaftlich arbeitenden Arztpraxis vorliegt und auch die Höhe des ärztlichen Reinertrags ${ }^{7}$ angemessen ist, dann ist zu prüfen, ob der durchschnittliche Aufwand für die Erbringung einer Leistung (Stückkosten bzw. Durchschnittskosten) stärker oder schwächer gestiegen ist als die durchschnittliche Vergütung je Leistungsmenge (Leistungspreis/Stückertrag) ${ }^{8}$ :

$\Delta \mathrm{t}\left(\frac{V_{t}}{L_{t}}-\frac{A_{t}}{L_{t}}\right) \lesseqgtr 0$

\section{V: Vergütung/Einnahmen \\ A: Aufwand \\ L: Leistungsmenge}

Der Orientierungswert würde demnach erhöht werden, wenn die Veränderung der Stückkosten oberhalb der Veränderung der Stückerträge liegt und

$3 \S 87 a$ Absatz 2 SGB V

4 Der Orientierungswert wurde rechnerisch durch die Division des auf das Jahr 2009 hochgerechneten Finanzvolumens durch die Leistungsmenge ermittelt (vgl. § 87c Absatz 1 SGB V [alt])

5 KBV (2013); Heinrich (2014)

6 Daneben einigten sich KBV und Krankenkassen noch auf eine weitere Stützung der hausärztlichen und fachärztlichen Grundversorgung in Höhe von 140 Mio. Euro bzw. ca. 0,5\%

7 Der Reinertrag entspricht dem Praxisüberschuss nach Abzug der Praxisaufwendungen von den Praxiseinnahmen vor Steuern; er ist damit mit dem Bruttoeinkommen eines abhängig Beschäftigten zu vergleichen.

8 Diese Modellstruktur geht auf das Gutachten der Fa. Prognos (2012) zurück; auf eine genaue Ausformulierung wird hier aus Vereinfachungsgründen verzichtet. Auch eine Diskussion der Mengenkomponente unterbleibt, da dies für die Gesamtargumentation nicht erforderlich ist; ein Problem bei der Umsetzung ergibt sich aufgrund der Unterschiede zwischen vereinbarter Leistungsmenge (Nettoleistungsbedarf) und abgerechneter Leistungsmenge (Bruttoleistungsbedarf), da die Krankenkassen nur die vereinbarte Leistungsmenge $\mathrm{zu}$ vergüten haben. Auch die Problematik des „Arztzahlrisikos" wird ausgeklammert, da eine Arztzahlzunahme nach Ansicht des Autors nicht von den Krankenkassen zu tragen ist. 
umgekehrt. Auf diesem Grundmodell aufbauend können Vorgaben des Gesetzgebers zur Anpassung des Orientierungswertes gemäß $\mathbb{S} 87$ Absatz $2 \mathrm{~g}$ SGB V, hierzu zählen die Veränderung der Investitions- und Betriebskosten, die Kostendegression und die Wirtschaftlichkeitsreserven, in die Kalkulation der Leistungspreise einbezogen werden. Wichtig ist aber die Berücksichtigung sämtlicher Einnahmenbestandteile, da es ansonsten zu einer Doppelfinanzierung der Praxiskosten kommen würde. Durch den Bezug auf die Leistungsmenge wird überdies sichergestellt, dass der durchschnittliche Reinertrag der Ärztinnen und Ärzte je zusätzlichem Leistungspunkt ansteigt und so eine angemessene Vergütung (c. p.) gewährleistet werden kann. Gleichfalls sind das Witschaftlichkeitsprinzip und die bereits vereinbarte budgetierte Gesamtvergütung bei der Anpassung des Orientierungswertes zu beachten.

Die Auffassung, ${ }^{9}$ dass es zu einem Preisverfall der ärztlichen Leistungen kommt, wenn der Orientierungswert nicht jährlich angehoben wird, orientiert sich an einem deutlich reduzierten Modell, das ausschließlich die Preisentwicklung der Praxiskosten und den sogenannten Arztlohn der Anpassung des Orientierungswertes zugrunde legt. Diese Sichtweise blendet die Gesamtvergütungsentwicklung aus und sieht die Funktion des Orientierungswertes lediglich im Inflationsausgleich und der Einkommensfortschreibung.

\section{Preis ist nicht gleich Preis: Der Preis je ärztliche Leistung hat sich in den letzten Jahren deutlich erhöht}

Auf den ersten Blick scheint es ja auch tatsächlich so zu sein, dass die geringe Anpassung des Orientierungswertes der letzten Jahre - seit 2009 wurde erstmals für das Jahr 2013 eine Anpassung von $0,9 \%$ vorgenommen - und damit die Bewertung der Leistungen in der EuroGebührenordnung nicht zu einem In- flationsausgleich geführt hat. Dies ist aber falsch, denn entscheidend für die Einschätzung, ob eine Kostendeckung und damit ein Inflationsausgleich stattgefunden hat, ist der tatsächlich abgerechnete Leistungspreis, der sämtliche Einnahmen bezogen auf die Leistungsmenge umfasst. Diese Unterscheidung resultiert aus der besonderen Funktion des Orientierungswertes, der zwar als zentraler Leistungspreis die vereinbarte Gesamtvergütung mitbestimmt, aber letztere auch von dem vereinbarten Behandlungsbedarf und weiteren Vergütungsvereinbaungen wie z. B. Preiszuschlägen abhängt. Konkret bedeutet dies, dass es zu einem Preisanstieg je Leistung kommt, wenn die Vergütung bzw. die Einnahmen stärker ansteigen als die abgerechnete Leistungsmenge. Zwischen 2008 und 2013 stieg der tatsächliche Leistungspreis um 8\% (Abbildung 1), da die vertragsärztliche Vergütung bundesweit mit rd. 16\% - in den neuen Bundesländern sogar 28\% - deutlicher anstieg als die Leistungsmenge, und übertraf damit deutlich den Orientierungswertanstieg von $0,9 \%$; die Verbraucherpreise erhöhten sich im gleichen Zeitraum um rd. 7\%. Genau genommen gibt es also zwei Preise, einerseits den Orientierungswert, der die Höhe der Gesamtvergütung bestimmt, und andererseits den tatsächlich erhaltenen Leistungspreis der Arztpraxis. Dies macht deutlich, dass erstens die Leistungspreise ansteigen können, auch wenn der Orientierungswert nicht angehoben wird und zweitens sämtliche Einnahmen in die Kalkulation einbezogen werden müssen.

\section{Berücksichtigung der Kostendegression (economies of scales) und der Arbeitsproduktivität}

Für die Anpassung des Orientierungswertes sieht der Gesetzgeber die Berücksichtigung der Kostendegression gemäß \$ 87a Absatz 2g Nr. 3 SGB V vor. Dahinter steht die Überlegung der „economies of scales“, dass mit steigender Leistungsmenge aufgrund eines vorhandenen Fixkostenanteils die Durchschnittskosten sinken (Fixkostendegression). Umstritten ist nun nicht das Prinzip, aber die Höhe des Fixkostenanteils, da ein hoher Anteil dazu führt, dass die Durchschnittskosten bei einem Leistungsmengenanstieg deutlicher sinken und damit einen Teil des Preisanstiegs kompensieren. In der be-

9 vgl. Stillfried/Czihal (2014), KBV (2014) S. 13 und Heinrich (2014) 
triebswirtschaftlichen $\mathrm{Li}^{-}$ teratur wird Dienstleistungsunternehmen ${ }^{10}$ ein eher hoher Fixkostenanteil zugeordnet, der daraus resultiert, dass im Gegensatz zu Industrieprodukten aufgrund fehlender Lagerfähigkeit und unregelmäßiger Nachfrage bestimmte Kapazitäten vorgehalten werden, um gegebenenfalls auch Nachfragespitzen abdecken zu können. Entsprechend weisen die wenigen Studien $^{11}$ zur Kostenstruktur in Arztpraxen auf hohe Fixkostenanteile hin. Ein guter Hinweis für erhebliche Fixkosten lässt sich auch aus dem deutlichen Arbeitsproduktivitätszuwachs beim Praxispersonal (Vollzeitäquivalente) ablesen, der in den letzten 10 Jahren bezogen auf die GKV-Leistungsmenge um $20 \%$ angestiegen ist.

Letztendlich wird man aufgrund der Datenlage und der methodischen Messprobleme den jeweiligen Fixkostenanteil normativ festlegen müssen. Neben der Fixkostendegression ist auch die Arbeitsproduktivität von Bedeutung. Steigt die Arbeitsproduktivität, d. h. die Leistungsmenge bzw. die Vergütung bezogen auf den Arzt, den Praxisinhaber oder die Arztpraxis nimmt zu, dann würden auch die Leistungspreise bei einer entsprechend elastischen Nachfrage sinken. Empirisch ist auch bei Arztpraxen eine deutliche Zunahme der Arbeitsproduktivität bezogen auf die ärztliche Arbeitszeitkapazität um 12\% zwischen 2008 und 2012 zu beobachten (Abbildung 2). Für die ärztliche Tätigkeit ist dieser Steigerung der Arbeitsproduktivität aufgrund fester Leistungspreise nicht unmittelbar umsetzbar, so dass die Berücksichtigung steigender Arbeitsproduktivität bei der Anpassung des Orientierungswertes erwogen werden sollte. Mit einer Verminderung der Innovationsanreize ist dabei nicht zu rechnen, denn Arztpraxen mit hoher Arbeitsproduktivität und hoher Auslastung werden weiterhin einen überdurchschnittlichen Reinertrag realisieren können.

\section{Auch die ärztliche Arbeitszeit wurde aufgewertet - mit einem unschönen Effekt für die Versorgung}

$\mathrm{Zu}$ beobachten ist seit mehreren Jahren ein Rückgang der durchschnittlichen ärztlichen Arbeitszeit auf mittlerweile 45 Wochenarbeitsstunden, ${ }^{12}$ die damit unterhalb der Normarbeitszeit des EBM von 51 Wochenarbeitsstunden liegt. Diese Entwicklung ging einher mit deutlichen Vergütungserhöhungen, was den Schluss nahe legt, dass finanzielle Anreize nicht automatisch zu einem verstärkten Arbeitsangebot führen. In der ökonomischen Literatur wird dieser Zusammenhang, dass die hier beobachtete Abnahme des zeitlichen Arbeitsangebotes je Arzt mit einer gleichzeitigen Einkommenserhöhung zusammenfällt, als „backward-bending“-Effekt bezeichnet. In einer Studie der Weltbank wird dieses Thema ausgeführt: „The policy implications of the conclusion are that increasing doctor's wages should not be expected to increase their hours worked".13 Zwar trifft dies sicher nicht für alle Ärzte gleichermaßen zu, es kann aber offensichtlich nicht von vornherein davon ausgegangen werden, dass finan-

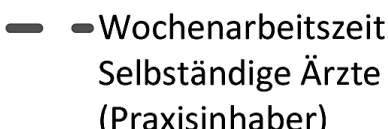

(Praxisinhaber)

Arbeitsproduktivität
bezogen auf die
Leistungsmenge in
Punkten je
Praxisinhaber

zielle Anreize die ambulante Versorgung der Patienten, z. B. Verringerung der Wartezeiten, verbessern - eher scheint bisher das Gegenteil eingetreten zu sein.

\section{Die Wirtschaftlichkeit der Arztpraxen messen}

Neben der Kostendegression führt das Gesetz die Möglichkeit zur Ausschöpfung von Wirtschaftlichkeitsreserven gemäß $\int 87$ Absatz 2g Nr. 2 SGB V an. Dieser Anpassungsfaktor wurde bislang entgegen anderslautender Aussagen ${ }^{14}$ nicht bei der Kalkulation des Orientierungswertes berücksichtigt. Es kann durchaus davon ausgegangen werden, dass ein erhebliches Wirtschaftlichkeitspotential durch vermehrte Kooperation und Konzentration von Arztpraxen sowie durch verbesserte Praxisorganisation bei ca. 85.000 Arztpraxen vorhanden ist. Verschiedene Konzepte hierzu werden gegenwärtig überprüft, doch liegt die Schwierigkeit darin, angemessene
10 Gerling et al (2004)
11 Gunning/Sickles (2011) gehen von einem Fixkostenanteil von $66 \%$ aus.
12 Statistisches Bundesamt (Mikrozensus)
13 Preker et al 2007: S. 197
14 KBV (2014): S. 13 


\section{Abbildung 3: Reinerträge 2011 nach Arztgruppen unterscheiden sich deutlich}

Angaben Reinertrag je Praxisinhaber in Tausend Euro 2011 (o. MVZ); Gesamteinnahmen einschl. Einnahmen aus der Privatpraxis; Quelle: Statistisches Bundesamt 2013

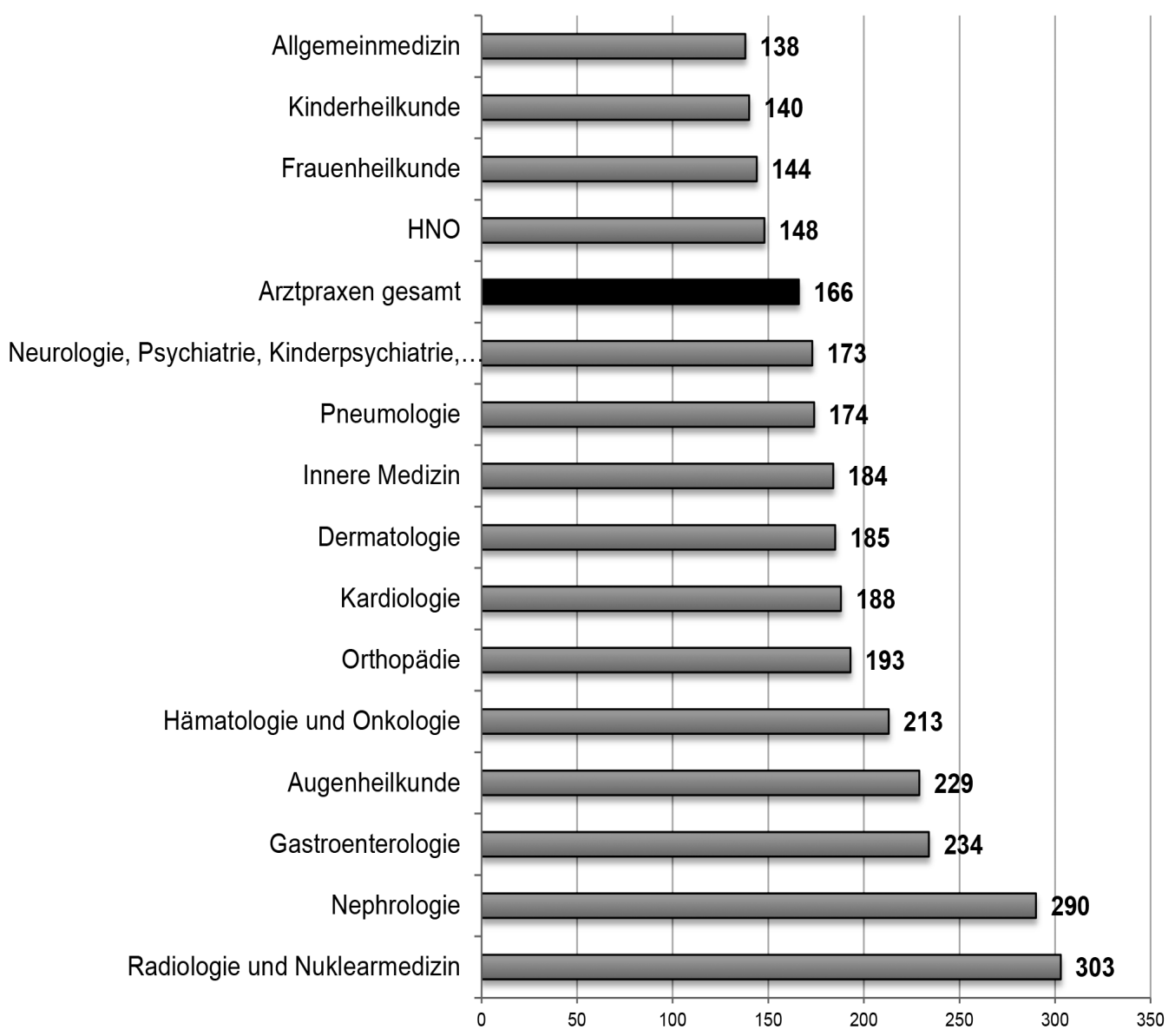

Anmerkung: Das Statistisches Bundesamt weist für einige internistische Schwerpunkte (Gastroenterologie, Kardiologie, Hämatologie) keinen Reinertrag aus, da die Ergebnisse als unsicher eingeschätzt werden; allerdings sind ausreichend Angaben vorhanden, die eine eigene Berechnung ermöglichen. Die Ergebnisse für die Arztgruppen Augenheilkunde, Neurolgie, Psychiatrie, Kinderpsychiatrie, Psychotherapie sind It. Statistischem Bundesamt statistisch relativ unsicher.

Selbstverständnis des Arztes als freiberuflicher Unternehmer nicht vereinbar. Hinzu kommt noch, dass die Höhe des Arztlohns von der Anzahl der Ärzte abhängt und die Krankenkassen dieses „Arztzahlrisiko“ nicht zu tragen haben. Offensichtlich hat dies auch den Gesetzgeber bei der Abfassung des $\mathbb{8} 87$ Absatz $2 \mathrm{~g}$ SGB V bewogen, neben den Praxiskosten auf die Anpassung des Arztlohns oder die Berücksichtigung der ärztlichen Arbeitszeit zu verzichten. Trotzdem ist es unbefriedigend, den Arztlohn vollkommen aus der Betrachtung herauszuhalten. Um den Arztlohn einzubeziehen, müsste der tatsächlich erreichte vergleichbare Gewinn in regelmäßigen Abständen überprüft werden und erst dann könnte über eine weitere Anpassung entschieden werden; denn selbst ohne Anpassung des Orientierungswertes - wie bereits vorher dargestellt wurde - ist ein steigender Praxisgewinn und damit ein höherer Preis für die ärztliche Leistung realisierbar.

Bislang wurde das ärztliche Einkommensniveau

empirische Datengrundlagen für einen Wirtschaftlichkeitsvergleich zu erhalten, die gleichfalls auch Besonderheiten der Versorgungsstruktur hinreichend berücksichtigen. Letztendlich obliegt es dem Bewertungsausschuss, hier ein angemessenes Gleichgewicht zwischen Berücksichtigung von Wirtschaftlichkeitsreserven und Versorgungssicherstellung zu vereinbaren. Keinesfalls kann es aber darum gehen, die Anforderungen an das Wirtschaftlichkeitsmaß durch eine Differenzierungstiefe bei den Praxisbesonderheiten soweit zu erhöhen, dass auf Basis der vorhandenen Datengrundlagen keine Entscheidung mehr möglich ist. Folglich würde man sich dann von jeglicher Messung der Wirtschaftlichkeit in Arztpraxen verabschieden.

\section{Die jährliche Fortschreibung des Arztlohns ist nicht sinnvoll}

Einen besonderen Stellenwert bei der jährlichen Anpassung des Orientierungswertes nimmt die Frage einer angemessenen Einbeziehung des sogenannten Arztlohnes ein, der als Vergütung für den Praxisinhaber angesehen wird. Für die Bestimmung des Orientierungswertes ist eine Einbeziehung eines Arztlohns als Kostenfaktor vom Gesetzgeber nicht vorgesehen. Der Arztlohn ist kein originärer Inputfaktor, sondern ist erst im Nachhinein als realisierter Gewinn aus der gesamten Unternehmertätigkeit des Arztes ermittelbar. Insofern wäre eine jährliche Fortschreibung des Arztlohns z. B. in Form eines Tariflohnes eines abhängig Beschäftigten eigentlich auch mit dem in die Anpassung des Orientierungswertes nicht einbezogen. ${ }^{15}$ Tatsächlich ist die konkrete Einbeziehung auch nicht einfach. Als Vergleichseinkommen wird der vom Bewertungsausschuss für die Bewertung der ärztlichen Gebührenpositionen im EBM festgesetzte sogenannte kalkulatorische Arztlohn angesehen, der sich an den möglichen Opportunitätskosten in Höhe des vergleichbaren Gehaltes eines Krankenhausarztes orientiert, wobei aber einige Besonderheiten zu berücksichtigen sind.

15 Bemerkenswerterweise verweisen gerade von Stillfried/Czihal (2013) auf das Referenzeinkommen Arztlohn als Maßstab für die Einbeziehung des Arzteinkommens in die Anpassung des Orientierungswertes und kritisieren dabei den GKV-Spitzenverband, obwohl die KBV dieses Konzept im Bewertungsausschuss ablehnte. 
Der kalkulatorische Arztlohn - seit 2008 in Höhe von 105 Tsd. Euro für eine nur über GKV-Einnahmen verfügende Vollzeitpraxis von 51 Wochenarbeitsstunden ${ }^{16}$ - war ursprünglich nur als Grundlage für eine betriebswirtschaftliche Kalkulation von Leistungen im EBM zwischen den Arztgruppen vereinbart worden, um eine bessere und rechtssichere Vergleichbarkeit der EBMLeistungen herzustellen. Da aber keine Arztpraxis nur über GKV-Einnahmen verfügt, handelt es sich beim kalkulatorischen Arztlohn um eine virtuelle Größe, die demnach für eine Opportunitätskostenbetrachtung in Bezug auf einen vergleichbaren Krankenhausarzt eingeschränkt aussagekräftig ist; denn der tatsächliche Praxisüberschuss übertrifft den kalkulatorischen Arztlohn bei weitem, so dass eine Erhöhung des Arztlohns den Abstand zwischen den Einkommen von Vertragsund Krankenhausärzten noch weiter vergrößern würde. ${ }^{17}$ Der tatsächliche Praxisüberschuss, der Reinertrag, ist allerdings als Vergleichsgröße gleichfalls problematisch, da dieser neben den GKV-Einnahmen auch die Einnahmen aus der Privatpraxis umfasst; mittlerweile hat diese Einkommensgröße mit durchschnittlich 166 Tsd. Euro (2011) je Praxisinhaber ein sehr hohes Niveau erreicht - wobei sich erhebliche Unterschiede zwischen den Arztgruppen ergeben (Abbildung 3) - und liegt damit zweifellos weit oberhalb der Gehälter langjähriger Fachärzte oder Oberärzte in Kliniken. Selbst die Ärzte in den neuen Bundesländern, die sich überwiegend durch GKV-Einnahmen finanzieren, erreichen mittlerweile Reinerträge in Höhe von 154 Tsd. Euro (Abbildung 4).

Wie haben sich nun aber die mit dem kalkulatorischen Arztlohn vergleichbaren Überschüsse in den letzten Jahren entwickelt? Bereits für das Jahr 2007 hatte das Institut des Bewertungsausschusses (InBA) ${ }^{18}$ errechnet, dass der kalkulatorische Arztlohn (2008) vorfristig erreicht worden war. Dabei ist das InBA von der konservativen Annahme eines um das 2,3fache höheren Preisniveaus der Privateinnahmen ausgegangen, was nicht unproblematisch ist, da auch ein unterschiedliches Ausgabenniveau und höhere ärztliche Arbeitszeiten für Privatpatienten unterstellt werden können. Geht man von dieser recht konservativen Annahme des InBA aus, dann liegt der so modifizierte Überschuss - bereinigt um den Preiseffekt der Privateinnahmen - für das Jahr 2011 bei 118 Tsd. Euro. Würde man relativ höhere Aufwendungen für die Behandlung von Privatpatienten z. B. von $10 \%$ berücksichtigen und die weiteren Vergütungssteigerungen bis 2013 einkalkulieren, so steigt dieser modifizierte Reinertrag je Praxisinhaber sogar auf knapp 130 Tsd. Euro im Jahr 2013 an. ${ }^{19}$ Auch hier zeigt sich, dass selbst ohne Anpassung des Orientierungswertes nicht nur die ärztlichen Reinerträge über die gesamten Einnahmen in den letzten Jahren angewachsen sind, sondern auch die Praxisüberschüsse auf Basis der GKVEinnahmen.

In der aktuellen Diskussion ${ }^{20}$ wird neben der Messung des Arztlohns als Vergleichseinkommen die zusätzliche „angemessene“ Einbeziehung eines schwer zu bestimmenden unternehmerischen Risikos gefordert. Angesichts der recht komfortablen Situation der Vertragsärzte, eine feste monatliche Abschlagszahlung von der Kassenärztlichen Vereinigung zu erhalten, dürfte dieses Risiko wohl eher als homöopathische Dosis auszumachen sein. Dies zeigt sich auch darin, dass das Insolvenzrisiko praktisch nicht vorhanden ist. ${ }^{21}$ Vielleicht wäre dann der Übergang in feste Angestelltenverhältnisse der ambulant tätigen Ärzte die konsequente und für die GKV günstigere Lösung, da auch viele junge Ärzte ohnehin kein ausgeprägtes Interesse mehr an einer Freiberuflichkeit zu haben scheinen.

16 Grundlage für die Festlegung durch den Erweiterten Bewertungsausschusses für 2008 war die Vergütung von Ärzten in Krankenhäusern, damals orientiert an BAT Ia. Vgl. Beschluss des Erweiterten Bewertungsausschusses in seiner 5. Sitzung am 11./12. Oktober 2007.

17 Interessanterweise wird für die Bewertung von Arztpraxen nur von einem Arztgehalt in Höhe von 76.000 Euro (2008) ab einem übertragbaren Umsatz von 240 Tsd. Euro im Jahr ausgegangen (Bundesärztekammer/ Kassenärztliche Bundesvereinigung (2008), S. A5).

18 Institut des Bewertungsausschusses (2010): S. 8

19 Eigene Berechnungen auf Basis der Ergebnisse des Statistischen Bundesamtes (2013)

20 KBV (2014): S. 13

21 Sparkassen-Finanzgruppe Branchendienst (2011): S. 35 


\section{Dynamisierung des Arztlohns und „cost disease“}

Obwohl die Entwicklung der ärztlichen Reinerträge eine Anhebung des Orientierungswertes nicht rechtfertigt, wird von Ärzteseite eine regelmäßige Erhöhung z. B. entsprechend der Tarifsteigerungen der Krankenhausärzte ${ }^{22}$ eingefordert. Eine solche Umsetzung hätte zur Folge, dass ca. 50\% der Orientierungswertanhebung nicht mehr im Bewertungsausschuss verhandelt werden, sondern von den Tarifabschlüssen, die der Marburger Bund in den letzten Jahren verhandelte, abhängen. Da der Marburger Bund seine Tarifforderungen auch mit den bereits erreichten hohen Einkommen der Niedergelassenen begründen kann, wird es zu einem gegenseitigen Hochschaukeln von ärztlicher Vergütung und den Tarifgehältern der Krankenhausärzte kommen. Ob die GKV diese Ausgabendynamik finanziell bewältigen kann, ist höchst fraglich.

Eine weiterer aktueller Vorschlag ${ }^{23}$ für eine jährliche Anpassung des Arztlohns sieht vor, neben einem Inflationsausgleich noch einen zusätzlichen Aufschlag in Form des allgemeinen Anstiegs der Arbeitsproduktivität der Volkswirtschaft einzubeziehen. Verwiesen wird auf die Überlegungen des amerikanischen Ökonomen William J. Baumol, der sich mit der Entwicklung des Dienstleistungssektors beschäftigte und hier den Begriff , cost disease“ (Kostenkrankheit $)^{24}$ prägte. Konkret befasste sich Baumol mit der Frage, warum die relativ unproduktiveren Sektoren wie Bildung, Kultur und Gesundheit einen stetig steigenden Anteil am Bruttosozialprodukt haben. Die Ursache liegt dabei nicht in einem höheren Wachstum der Löhne im Dienstleistungssektor, diese können sogar unterhalb der allgemeinen Nominallohnentwicklung verlaufen, sondern in der Veränderung der relativen Preise zwischen Dienstleistungen und kapitalintensiv produzierten Industriegütern. Diese Entwicklung ist in allen entwickelten Volkswirtschaften mehr oder weniger stark zu beobachten ${ }^{25}$, doch sind die Ursachen nicht ausschließlich auf die „cost disease“ zurückzuführen.
Im Übrigen findet sich bei Baumol keine mechanistische Handlungsempfehlung für eine Anpassung von Einkommen in produktiven und unproduktiven Sektoren, sondern er verweist auf vielfältige Wirkungszusammenhänge wie z. B. die Preiselastizitäten und Einkommenseffekte. Nach Baumol werden die Ausgaben in Dienstleistungssektoren zwar stetig wachsen, doch bedeutet dies nicht, dass deren „level of costs cannot be brought down" ${ }^{26}$. Baumol verweist Die jährliche Fortschreibung es Arztlohns würde nur zu einer Dynamisierung der ärztlichen Vergütung und u Preisverzerrungen im Vergleich zum Einkommen der Krankenhausärzte beitragen.

explizit darauf, dass auch im Gesundheitswesen Produktivitätsreserven vorhanden sind, z. B. durch Vermeidung medizinisch nicht wirksamer und damit unwirtschaftlicher Leistungsangebote, die zu Einsparungen und zu einem relativen Sinken der Preise auch im Gesundheitssektor führen können. Insofern bietet sich der Bezug auf die Baumolsche Kostenkrankheit für die Diskussion um den Orientierungswert nicht an, höchstens dahingehend, dass das Arzteinkommen dann nicht entsprechend der Tarifentwicklung der Krankenhausärzte weiterzuentwickeln wäre, sondern entweder entsprechend der Inflationsentwicklung oder der Entwicklung der bundesdurchschnittlichen Nominallöhne. Eine Anhebung oberhalb dieser Entwicklung wäre einkommens- und strukturpolitisch kaum begründbar, hätte u. U. negative Einflüsse auf das volkswirtschaftliche Wachstum ${ }^{27}$ und würde dazu führen, dass sich der Anteil der Gesundheitsausgaben am BIP stetig zulasten anderer Dienstleistungssektoren wie z. B. Bildung erhöht.

\section{Das derzeitige Verfahren zur Anpassung des Orientierungswertes}

Das im März 2013 im Bewertungsausschuss vereinbarte Verfahren zur Anpassung des Orientierungswertes ${ }^{28}$ stellte einen Kompromiss zwischen den unterschiedlichen Vorstellungen beider Seiten dar. So werden neben den gesetzlichen Anpassungsfaktoren auch die Veränderungen der Oberarztgehälter und der Vergütung in die Verhandlungen einbezogen. Die Anpassung des Orientierungswertes wird derzeit auf Basis einer jährlichen Veränderungsrate - für die Anpassung 2015 auf Basis der Veränderungsrate des Jahres 2013 gegenüber 2012 - vorgenommen. Grundlage für die Ermittlung der Veränderungsraten der Aufwendungen je Leistungspunkt (Durchschnittskosten) ist das vom InBA vorgeschlagene EBMKalkulationssystem, das sogenannte Standardbewertungssystem. Mit der Verbindung von EBM-Kalkulation und Anpassung des Orientierungswertes verband man die Vorstellung, dass eine gegenseitige Übertragbarkeit von Preiskalkulationen zwischen EBM und Orientierungswertanpassung möglich sei. Grundsätzlich können mit diesem Verfahren die Stückkosten auf Basis der im Standardbewertungssystem einbezogenen Leistungsmengen errechnet werden. Einige hiermit verbundene Probleme sind weiterhin in der Diskussion; hierzu zählen insbesondere die Einbeziehung der Anzahl der Ärzte ${ }^{29}$ oder von Arztpraxen sowie die Bewertung einer

\section{Stillfried/Czihal (2014)}

23 Stillfried/Czihal (2013); KBV (2014): S. 13

24 Die Analyse von Baumol ist aber nicht unumstritten (vgl. Cowen 1996)

25 Empirisch ist zwischen 2001 und 2010 allerdings zu beobachten, dass der „Anstieg der Arbeitsproduktivität in der Gesundheitswirtschaft mit 1,9\% deutlich über dem gesamtwirtschaftlichen Durschnitts von 1,6\%" lag (Schneider et al (2013), S. 12).

26 Baumol (2012): S. 154

27 Der positive Einfluss von Gesundheitsausgaben auf das wirtschaftliche Wachstum ist durchaus umstritten. Vgl. Dormont et al (2007); auch die Umverteilung zugunsten der ärztlichen Einkommen ist makroökonomisch als problematisch einzuschätzen, da dies die Sparrate erhöht und umgekehrt die Konsumquote senkt; auch die Beschäftigung beim Praxispersonal (Vollzeit) ist seit Jahren annähernd konstant.

28 Beschluss des Erweiterten Bewertungsausschusses in der 34. Sitzung am 19.03.2013

29 Mit der Einbeziehung der Anzahl der Arztpraxen wird durch die „,kalte Küche“ das Arztzahlrisiko auf die Krankenkassen übertragen, obwohl der Gesetzgeber dieses bereits im GKV-WSG ausdrücklich ausgeschlossen hat: Zentrales Ziel der Vergütungsreform 2009 war der Übergang des Morbiditätsrisikos auf die Krankenkassen, aber nicht die Finanzierung von „Mehrleistungen, die auf Grund einer Zunahme von Arztzahlen entstehen." 
sinkenden Auslastung von Arztpraxen. Sinkende Auslastungen bedeuten Effizienzverluste der Versorgung und widersprechen damit dem Wirtschaftlichkeitsprinzip. Mit dem Beschluss 2013 ${ }^{30}$ soll dieses Verfahren in Bezug auf seine Wirkungsweise hin überprüft werden.

\section{Schlussfolgerungen}

Die Reinerträge haben sich auch bei konstantem Orientierungswert und steigender Arztanzahl in den letzten Jahren deutlich erhöht, so dass eine „schleichende Abwertung der ärztlichen Arbeitszeit" und ein fehlender Inflationsausgleich für steigende Praxiskosten nicht zu erkennen sind. Um zukünftig eine wirtschaftlich angemessene Finanzierung der vertragsärztlichen Versorgung zu erreichen, ist eine sachgemäße Festlegung des Orientierungswertes von großer Bedeutung. Dies kann nur dadurch erreicht werden, dass sämtliche Einnahmen (Vergütungsbestandteile) ebenso in die Kalkulation eingezogen werden wie die preisliche Entwicklung der Praxiskosten. Der Gesetzgeber hat mit dem GKV-WSG die entscheidenden Anpassungsfaktoren, - Preisangleichung der Investitions- und Betriebskosten, Kostendegression und potentiellen Wirtschaftlichkeitsreserven-, benannt, die vom Bewertungsausschuss anzuwenden sind.

Die gute ökonomische Situation der Arztpraxen rechtfertigt eine Einbeziehung des Arztlohns in die Anpassung des Orientierungswertes ebenso wenig wie eine regelmäßige Anhebung auf Basis von Tarifabschlüssen der Krankenhausärzte oder allgemeinen Produktivitätssteigerungen. Die jährliche Fortschreibung des Arztlohns würde nur zu einer Dynamisierung der ärztlichen Vergütung und zu Preisverzerrungen im Vergleich zu den Einkommen der Krankenhausärzte beitragen. Inwieweit zukünftig eine Anpassung erforderlich sein wird, hängt von der Entwicklung aller für die ökonomische Situation der Arztpraxis relevanten Einflussfaktoren ab. Dies könnte beispielsweise dadurch geschehen, dass der Bewertungsausschuss in regelmäßigen Abständen - denkbar wäre eine Koppelung an den 4-Jahreszyklus der Kostenstrukturerhebung des Statistischen Bundesamtes -, die Höhe der Reinerträge bzw. der Praxisüberschüsse überprüft. Bei der zukünftigen Weiterentwicklung des Arztlohns sollten die makroökonomischen Rahmenbedingungen beachtet werden und nur eine moderate Einbeziehung gemessen an der allgemeinen Einkommensentwicklung stattfinden.
Der bisher im Bewertungsausschuss beschrittene Weg, unterschiedliche Aspekte der Anpassung des Orientierungswertes in einem Verfahren zu integrieren, entspricht den z. T. recht konträren Sichtweisen der Beteiligten. Eine stärkere Konkretisierung der gesetzlichen Regelungen wäre zwar hilfreich, doch könnte der Bewertungsausschuss auf Grundlage der derzeitigen Regelungen durchaus zu vernünftigen Ergebnissen kommen, die es ermöglichen, sowohl die ärztliche Vergütung ohne zusätzliche Belastungen der Beitragszahler angemessen weiterzuentwickeln als auch wirtschaftliche Aspekte zu berücksichtigen.

30 Vgl. Beschluss des Erweiterten Bewertungsausschusses auf seiner 37. Sitzung vom 25.09.2013 zur Festlegung gemäß § 87 Absatz 2e SGB V und Anpassung gemäß § 87 Absatz 2g SGB V des Orientierungswertes für das Jahr 2014

\section{Literatur} Baumol, W. J. (2012): The cost disease, New
Haven and London

Bundesärztekammer, Kassenärztliche Bundesvereinigung (2008); Hinweise zur Bewertung von Arztpraxen (Stand 9.9.2008), in: Deutsches Ärzteblatt, Jg. 105, 22. Dezember 2008, S A4 ff

Cowen, T. (1996): Why I do not believe in the cost-disease, in: Journal of cultural economics, Heft 20.3, S. $207 \mathrm{ff}$

Deutscher Bundestag (2006): Gesetzentwurf der Fraktionen der CDU/CSU und SPD, Entwurf eines Gesetzes zur Stärkung des Wettbewerbs in der gesetzlichen Krankenversicherung (GKVWettbewerbsstärkungsgesetz GKV-WSG), Drucksache 16/3100

Dormont, B. et al (2007): Health expenditure, longevity and growth, Limone sul Garda

Frielingsdorf, O. (2011): Überblick zur BGHgemäßen Festlegung des individuellen Arztlohnes bei Bewertung von Arzt-/

Zahnarztpraxen im Ehescheidungsverfahren, in: FamRZ 2011, Heft 24, S. $1911 \mathrm{ff}$

Gerling, P. et al (2004): Aktueller Stand der Kostenrechnung für den Dienstleistungsbereich in Theorie und Praxis, Beiträge zur Controlling-Forschung, Nr. 3 (Working Paper), Kaiserslautern
Gunning, T. und Sickles, R. (2011): A multi-product cost function for physician private practices, Journal of Productivity Analysis 35, S. $119 \mathrm{ff}$

Heinrich, D. (2014): Es muss wieder feste Preise geben, in: Gesundheit und Gesellschaft Heft 3, 17. Jahrgang, S. 26

Kaiser, P. (2014): Vertragsärztliche Gesamtvergütung, im: GKV-Lesezeichen 2014, „Neues bewerten - Bewährtes erneuern“, Berlin, S. $178 \mathrm{ff}$

KBV (2013): Erwartungen der niedergelassenen Ärzte und Psychotherapeuten an eine zukunftssichere Gesundheitspolitik - Position zur Sicherstellung der ambulanten Gesundheitsversorgung, Berlin

KBV (2014): Positionierung der Kassenärztlichen Bundesvereinigung zum Koalitionsvertrag von CDU, CSU und SPD zum Abschnitt Gesundheit und Pflege (18. Legislaturperiode), Berlin

Institut des Bewertungsausschusses (2010): Überprüfung der EBM-Kalkulationsgrundlagen, Berlin (unveröffentlichter Bericht)

Institut des Bewertungsausschuss (2013): Bericht über die vorbereitenden Arbeiten des Instituts des Bewertungsausschusses zur Entwicklung eines datengestützten Verfahrens zur regelmäßigen Anpassung des Orientie- rungswertes nach $\S 87$ Absatz ze SGB V (Stand 1. August 2013) (unveröffentlichter Bericht)

Preker, A. et al (2007): Public ends, private means, World Bank, Washington

Prognos (2012): Bestimmung des Orientierungswertes 2013 für die vertragsärztliche Vergütung, Basel

Schneider, M. et al (2013): Messung der Produktivitätsentwicklung der Gesundheitswirtschaft (Kurzfassung), Studie im Auftrag des Bundesministeriums für Wirtschaft und Technologie (BMWI), Augsburg, Dresden und Berlin

Sparkassen-Finanzgruppe Branchendienst (2011): Branchenreport Fachärzte 2011, Stuttgart Statistisches Bundesamt (2013): Einnahmen und Reinerträge von Kassenpraxen: Privateinnahmen machen den Unterschied, Pressemitteilung vom 4. Dezember 2013, Wiesbaden

Statistisches Bundesamt (2013): Kostenstruktur bei Arzt und Zahnarztpraxen sowie Praxen von psychologischen Psychotherapeuten 2011, Wiesbaden

Von Stillfried, D. und Czihal, T. (2014): Ärztliche Arbeitszeit beim Orientierungswert berücksichtigen, in: Deutsches Ärzteblatt, Jg. 111, Heft 4, 24. Januar 2014, S. A 114 ff

ZI-Praxis-Panel (2013): Jahresbericht 2012, Berlin 